\title{
PROPUESTA DE AUTOMATIZACIÓN EN BODEGA DE PRODUCTO TERMINADO EN INDUSTRIA MANUFACTURERA DE PRODUCTOS DE HIGIENE PERSONAL EN COSTA RICA
}

Proposed warehouse automation in the finished product in manufacturing products personal hygiene in Costa Rica

\section{Esteban Pérez López ${ }^{1}$}

\begin{abstract}
Resumen
El presente artículo presenta la propuesta de automatización para la sección de bodega de producto terminado y área de entarimado de la empresa Kimberly Clark, donde se hace la selección de producto, inventariado, paletizado y entarimado del producto final. Se propuso automatizar parte de dicho proceso por medio de la lectura del código de barras de sus productos y la distribución de los mismos por canales específicos donde el operador humano intervenga hasta la etapa final de acomodo en tarimas para el paletizado, de manera que el personal no tenga que estar realizando trabajos repetitivos y desgastantes; y así se pueda llevar un control de inventario minucioso de forma automática. La propuesta se plantea a la planta de de la compañía en San Antonio de Belén de Heredia, Costa Rica.
\end{abstract}

Palabras Clave: automatización, industria, sistema flexible, sistemas modernos, manufactura, robótica.

\begin{abstract}
This article presents the proposal automation section finished product warehouse area parquet Kimberly Clark, where product selection is made, inventory, palletizing and parquet final product. It is proposed to automate part of the process by reading the bar code of their products and distributing them to specific channels where the human operator intervention until the final stage of accommodation in flooring for palletizing, so that staff not have to be performing repetitive and exhausting work; and so you can keep track of detailed inventory automatically. The proposal poses to the plant in San Antonio de Belén, Heredia, Costa Rica.
\end{abstract}

1. Máster en Sistemas Modernos de Manufactura y Bach. en Laboratorista Químico. Profesor UCR-Recinto Grecia. estebanperezlopez@gmail.com 
Keywords: automation, industry, flexible systems, modern systems, manufacturing, robotics.

\section{Introducción}

En el inicio del camino de la automatización, las primeras máquinas simples sustituían una forma de esfuerzo en otra forma que fueran manejadas por el ser humano, tal como levantar un peso pesado con sistema de poleas o con una palanca. Posteriormente las máquinas fueron capaces de sustituir formas naturales de energía renovable, tales como el viento, mareas, o un flujo de agua por energía humana.

La parte más visible de la automatización actual puede ser la robótica industrial. Algunas ventajas son repetitividad, control de calidad más estrecho, mayor eficiencia, integración con sistemas empresariales, incremento de productividad y reducción de trabajo. Algunas desventajas son requerimientos de un gran capital, decremento severo en la flexibilidad, y un incremento en la dependencia del mantenimiento y reparación. Por ejemplo, Japón ha tenido necesidad de retirar muchos de sus robots industriales cuando encontraron que eran incapaces de adaptarse a los cambios dramáticos de los requerimientos de producción y no eran capaces de justificar sus altos costos iniciales.

La producción a gran escala involucra tareas repetitivas, donde se debe mantener, además, un conjunto de magnitudes (por ejemplo la presión, la temperatura, etc.) dentro de márgenes preestablecidos. La aplicación de los dispositivos electromecánicos y electrónicos en el área industrial permitió automatizar las tareas repetitivas, aumentando así los niveles de producción, y controlar las magnitudes físicas en forma más precisa (Daneri, 2008).

Para mediados del siglo XX, la automatización había existido por muchos años en una escala pequeña, utilizando mecanismos simples para automatizar tareas sencillas de manufactura. Sin embargo el concepto solamente llego a ser realmente práctico con la adición (y evolución) de las computadoras digitales, cuya flexibilidad permitió manejar cualquier clase de tarea. Las computadoras digitales con la combinación requerida de velocidad, poder de cómputo, precio y tamaño empezaron a aparecer en la década de 1960. Antes de ese tiempo, las computadoras industriales eran exclusivamente computadoras analógicas y computadoras híbridas. Desde entonces las computadoras digitales tomaron el control de la mayoría de las tareas simples, repetitivas, tareas semi-especializadas y especializadas, con algunas excepciones notables en la producción e inspección de alimentos.

Según Arbones (2009), un sistema automatizado es un conjunto que, después de haber recibido instrucciones suministradas por un operador, decide y actúa, sustituyendo así al hombre. Tal 
sustitución produce mayor rapidez de ejecución, mejor regulación de los resultados y evita al hombre las tareas penosas y repetitivas.

El trabajo se realizó en Kimberly Clark, la cual es una empresa multinacional dedicada a la fabricación de productos higiénicos de papel y de cuidado personal con reconocidas marcas como: Scott, Kleenex, Huggies, Kotex, Scribe y productos institucionales.

\section{Marco teórico}

La historia de la automatización industrial está caracterizada por periodos de cambios bruscos en los métodos populares, según menciona Craig (2006). Ya sea como una causa o como un efecto, dichos periodos de cambio en las técnicas de automatización parecen estar estrechamente ligados con la economía mundial. El uso del robot industrial, que se identificó como dispositivo único en la década de 1960, junto con los sistemas de diseño asistido por computadora (CAD) y manufactura asistida por computadora (CAM), caracteriza las tendencias más recientes en la automatización del proceso de manufactura.

Pardo (2012) menciona que en el campo de la producción industrial, la automatización se ha convertido en una herramienta de trabajo necesaria e indispensable para optimizar los procesos productivos y aumentar la competitividad. Por tanto, la automatización no tiene otra misión que la de lograr un valor añadido en los productos manufacturados, variando sus características y llevando a cabo una transformación de materias o bienes hasta llegar a un producto acabado. La Automatización Industrial fue un término acuñado por un ingeniero de la Ford Motor Company , que se utiliza para describir una amplia variedad de sistemas en los que existe una sustitución en un proceso del operador humano, su esfuerzo e inteligencia, por dispositivos eléctricos, mecánicos, neumáticos, informáticos, etc. En términos generales, la automatización se puede definir como una tecnología referida a procesos controlados por medio de instrucciones programadas, junto a sistemas de control retroalimentados capaces de realizar ciclos más o menos complejos de operaciones que se repiten para garantizar la correcta ejecución de dichos procesos; que se sintetiza en la expresión: "the sulting system is capable of operating without human intervention" -el sistema resultante es capaz de funcionar sin intervención humana-.

Para Hernández (2012), las industrias también se han visto afectadas por el fenómeno de la modernización, con el uso de la fabricación asistida por ordenador o el software de ingeniería asistida por ordenador. Además, se ha aumentado el uso de la automatización y los robots, las máquinas de inspección visual, el control a tiempo real de la producción, los sistemas avanzados de fabricación y el modelado y recreación virtual de procesos y fábricas enteras, mediante software de simulación de producción asistida por ordenador. 
Una de las principales razones en el uso de robots industriales es la reducción en el costo de operación. Además los robots no solo se están volviendo más baratos sino también más efectivos, más rápidos, precisos y flexibles. A medida que los robots se vuelvan más efectivos en cuanto al costo por sus tareas, y a medida que aumente el costo por mano de obra humana, habrá más trabajos que se convierten en candidatos para la automatización robótica (Craig, 2006).

La Automatización Industrial implementa sistemas o elementos computarizados para controlar maquinarias y/o procesos industriales sustituyendo a operadores humanos. Es decir que cuando se habla de sistemas industriales de automatización, se refiere a aquellos elementos que permiten obviar o sustituir total o parcialmente la intervención humana en los procesos industriales, estando constituidos por sistemas cableados o programados principalmente (Gallardo, 2013).

García (2005) menciona que es la creciente necesidad de mejorar la productividad, lo que ocasiona que la automatización de los procesos industriales se convierta en una de las prioridades más acuciantes de las empresas de fabricación de hoy en día.

Con la automatización industrial la mano de obra se sustituye por dispositivos autónomos que controlan las maniobras y manipulaciones de materia prima encaminada a la obtención de los diversos productos demandados por la sociedad día con día (Domingo, 2003).

Se debe tener en cuenta que, existen muchos trabajos donde no existe riesgo inmediato de la automatización. Ningún dispositivo ha sido inventado que pueda competir contra el ojo humano para la precisión y certeza en muchas tareas; tampoco el oído humano. El más inútil de los seres humanos puede identificar y distinguir mayor cantidad de esencias que cualquier dispositivo automático. Las habilidades para el patrón de reconocimiento humano, reconocimiento de lenguaje y producción de lenguaje se encuentran más allá de cualquier expectativa de los ingenieros de automatización.

Computadoras especializadas, son utilizadas para leer entradas de campo a través de sensores y con base a su programa, generar salidas hacia el campo a través de actuadores. Esto conduce para controlar acciones precisas que permitan un control estrecho de cualquier proceso industrial. (Se temía que estos dispositivos fueran vulnerables al error del año 2000, con consecuencias catastróficas, ya que son tan comunes dentro del mundo de la industria).

Existen dos tipos distintos: DCS o Sistema de Control Distribuido, y PLC o Controlador Lógico Programable. El primero era antiguamente orientado a procesos de tipo análogos, mientras que el segundo se utilizaba en procesos de tipo discreto (ceros y unos). Actualmente ambos equipos se parecen cada vez más, y cualquiera de los dos puede ser utilizado en todo tipo de procesos. 
Como indica Daneri (2008), un PLC permite controlar un proceso o realizar una secuencia de acciones de manera automática a partir de un programa definido por el usuario. Esto lo realiza ejecutando en forma cíclica una secuencia de instrucciones que, a partir de la información que llega a sus entradas desde los sensores, deciden cuándo conmutar sus salidas, donde se encuentran conectados los actuadores. Las partes fundamentales que componen un PLC son la unidad central de proceso o CPU, y las interfaces de entrada y salida. La CPU es el cerebro del PLC y está formada por el procesador y la memoria. El procesador se encarga de ejecutar el programa escrito por el usuario que se encuentra almacenado en la memoria. Además, el procesador se comunica con el exterior mediante sus puertos de comunicación y realiza funciones de autodiagnóstico.

La aparición de los autómatas programables (PLC, Programmable Logic Controllers) permitió reducir en gran medida la cantidad de material necesario para conseguir controlar una máquina; los controles se programan en su interior y las modificaciones de funcionamiento no significan necesariamente cambios físicos y, además, el tiempo necesario para el mantenimiento se reduce (Rodríguez, 2008).

Las interfaces Hombre-Máquina $(\mathrm{HMI})$ o interfaces Hombre-Computadora $(\mathrm{CHI})$, formalmente conocidas como interfaces Hombre-Máquina, son comúnmente empleadas para comunicarse con los PLCs y otras computadoras, para labores tales como introducir y monitorear temperaturas o presiones para controles automáticos o respuesta a mensajes de alarma. El personal de servicio que monitorea y controla estas interfaces son conocidos como ingenieros de estación.

Otra forma de automatización que involucra computadoras es la prueba de automatización, donde las computadoras controlan un equipo de prueba automático que es programado para simular seres humanos que prueban manualmente una aplicación. Esto es acompañado por lo general de herramientas automáticas para generar instrucciones especiales (escritas como programas de computadora) que direccionan al equipo automático en prueba en la dirección exacta para terminar las pruebas.

La automatización como una disciplina de la ingeniería es más amplia que un mero sistema de control, abarca la instrumentación industrial, que incluye los sensores y transmisores de campo, los sistemas de control y supervisión, los sistemas de transmisión y recolección de datos y las aplicaciones de software en tiempo real para supervisar y controlar las operaciones de plantas o procesos industriales. 


\section{Características y propiedades de materiales para automatismos industriales}

Un aspecto relevante a la hora de definir la automatización requerida, son los materiales empleados ya que deben ser los apropiados y cuya normativa vigente lo permita. Para ello es necesario conocer las características, propiedades y aplicaciones de la materia prima disponible en el mercado para poder realizar la instalación con los materiales adecuados.

Rodríguez (2014), menciona las siguientes características que deben cumplir los materiales a utilizar en automatismos industriales:

- Adherencia: capacidad para que se produzca la unión entre dos o más cuerpos en contacto.

- Compresibilidad: capacidad de los cuerpos a ser comprimibles, es decir, reducir su volumen conservando su masa.

- Dilatabilidad: propiedad de los cuerpos de de extender, alargar u ocupar más volumen.

- Elasticidad: propiedad de los cuerpos para recuperar su longitud y forma originales al interrumpir la acción que los alteraba.

- Fatiga: pérdida de resistencia mecánica de un material al ser sometido largamente a esfuerzos repetidos.

- Plasticidad: capacidad que tienen los cuerpos a cambiar su forma y conservarla tras cesar la acción que lo produjo.

- Porosidad: propiedad que tienen los cuerpos sólidos de poseer cavidades entre las partículas o moléculas.

- Solubilidad: capacidad que tienen los cuerpos sólidos o espesos para disolverse o separarse las partículas o moléculas en un medio líquido logrando una mezcla homogénea.

De igual manera Rodríguez (2014), indica que los materiales a emplear en automatización industrial, deben tener propiedades especiales como:

- Conductividad: propiedad que tienen los cuerpos para transmitir el calor y la electricidad. Es característica de cada material y varía con la temperatura. En general, la conductividad eléctrica de los metales disminuye con la temperatura.

- Resistividad: es la inversa a la conductividad, siendo la propiedad que tienen los cuerpos para oponerse a transmitir el calor y la electricidad. Es característica de cada material y varía con la temperatura. En general la resistividad eléctrica para los metales aumenta con la temperatura. 
- Ductilidad: es la capacidad de algunos metales a ser deformados mecánicamente, moldeados, o extenderse con facilidad en frío sin romperse, extendiéndose en alambres o hilos.

- Dureza es la propiedad de los cuerpos de ofrecer resistencia a ser rayados.

- Fragilidad: capacidad de los cuerpos sólidos a ser quebrados o que se rompan con facilidad.

- Magnetización: propiedad de algunos cuerpos a poseer propiedades magnéticas o alta permeabilidad de imantación, atrayendo al hierro, el acero, y en grado menor otros cuerpos.

- Maleabilidad: propiedad de algunos metales a ser extensibles en planchas o láminas.

- Masa: es una magnitud que indica la cantidad de materia que hay en los cuerpos. Depende del material.

- Tenacidad: capacidad que tienen los cuerpos a oponer resistencia a deformarse o romperse.

- Volumen: propiedad que indica el espacio que ocupa un cuerpo, material o sustancia.

- Densidad: relación entre la masa y el volumen que ocupa un cuerpo. Es una propiedad característica de todos los cuerpos, pudiendo identificar distintos materiales y sustancias.

\section{Ventajas y desventajas de la automatización industrial}

En general, la automatización industrial pretende incrementar la competitividad de la industria, esta involucra ciertas desventajas, las cuales habrá que sopesar, junto con sus ventajas, a la hora de decidir si resulta conveniente embarcarse en un proceso de modernización (Anónimo, s.f.).

\section{Ventajas}

1. Aumento de la productividad: incremento de la cantidad de producto por unidad de tiempo.

2. Reducción de costos laborales: disminución de los costos de producción, especialmente los asociados a mano de obra.

3. Mejor calidad de producto.

4. Estabilización de las características del producto dentro de las especificaciones requeridas por los clientes.

5. Reducción de los costos por reprocesamiento de productos rechazados por el departamento de control de calidad. Esto es una consecuencia directa del punto previo.

6. Reducción de costos por consumo de energía: los sistemas automatizados pueden incluir un programa de minimización del consumo energético. 
7. Aumento de la seguridad laboral: las máquinas automatizadas pueden realizar aquellas labores dentro del proceso que, por su naturaleza riesgosa, ponen en peligro la salud de los trabajadores.

8. Dignificación del trabajo humano: al sustituir al operador humano por una máquina, este queda disponible para otras actividades en donde ponga en juego un mayor conjunto de sus posibilidades como ser humano y no con tareas repetitivas o aburridas.

9. Se han diseñado máquinas automatizadas que pueden desempeñarse en actividades dentro del proceso de producción que el hombre no está en capacidad de realizar, bien sea porque los requerimientos exceden su fuerza física, su destreza manual o su capacidad de atención , estas máquinas realizan tareas las veinticuatro horas del día, con muy pocas interrupciones.

10. Flexibilidad de producción: un sistema automatizado puede reducir apreciablemente los tiempos que se consumen elaborado una gama de productos.

11. Integración: los nuevos equipos y dispositivos empleados en la automatización industrial permiten su interconexión en redes de comunicación de datos, de tal forma que toda la planta pueden estar integrada dentro de una extensa red (similar a una red de computadoras), que abarque desde el nivel más bajo (detectores ubicados al nivel de las maquinas de producción), hasta los más altos (gerencias de producción, mantenimiento, gerencia general entre otros) inclusive mediante la conexión a internet, se puede tener acceso remoto a la planta y detectar su estado actual, nivel de producción, cuáles maquinarias están en actividad o paradas entre otras.

\section{Desventajas}

1. Resistencia al cambio: los operadores de planta e incluso los gerentes, a menudo ven los procesos basados en una mayor automatización, con un cierto rechazo inicial. Este puede conducir incluso a la pérdida total del nuevo sistema automatizado y la vuelta al antiguo.

2. Falta de conocimientos: resulta difícil (incluso para los expertos) mantenerse al día con las innovaciones de la automatización moderna. Como consecuencia, los gerentes encargados de la tomas de decisiones finales en cuanto a la adquisición de nuevos equipos o modernización de los mismos, muchas veces no están al tanto de los últimos desarrollos y de las posibilidades y ventajas que estos pueden aportar a sus procesos productivos.

3. Inversión inicial elevada: dada su sofisticación, los equipos y maquinarias automatizadas suelen tener un costo mayor, lo cual, a veces puede hacer injustificable un proceso de automatización. 
4. Ausencia de personal capacitado o que pueda ser entrenado satisfactoriamente en estas nuevas tecnologías.

5. Si se dispone de personal capaz de adaptarse a los nuevos equipos automatizados, siempre se tendrá la necesidad de capacitarlo.

6. Dependencia tecnológica: al ser más sofisticada la maquinaria, el productor puede verse obligado a mantener contratos exclusivos de mantenimiento con el proveedor de la tecnología.

7. Obsolescencia: la tecnología de automatización vive dentro de un rápido y constante proceso de innovación y mejoramiento. Por lo tanto, una costosa inversión en hoy, con la cual adquirimos la última tecnología disponible (haciéndonos altamente competitivos), puede quedar totalmente obsoleta a la vuelta de diez años e incluso menos.

8. Escasez de recurso humano especializado: resulta difícil conseguir personal especialistas en automatización en el mercado de trabajo, especialmente en algunos países.

9. Limitaciones de las maquinas automatizadas frente al operador humano: a pesar de todos los desarrollos tecnológicos, aún persisten áreas en donde el hombre supera ampliamente a la máquina. Por ejemplo, el ser humano aprende mucho mejor de la experiencia, es capaz de tomar decisiones acertadas, incluso sin contar con la totalidad de información.

10. Imposibilidad de automatizar debido a la ausencia de detectores adecuados para la aplicación: todavía existen actividades en el campo de la sensórica que permanecen exclusivamente reservadas para los seres humanos, por ejemplo, la detección crítica de olores y sabores, acabado final de piezas artesanales, estado de suciedad del producto, etc.

\section{Metodología}

Para la ejecución del presente estudio se evaluó in situ durante sesenta días la situación en la bodega de producto terminado en la empresa Kimberly Clark, y se ofrece una solución de automatización industrial, proponiendo un proyecto de interés a la compañía, que le haga más eficiente en su sector a automatizar, sin hacerle incurrir en inversiones desproporcionadas, en caso de que deseen implementar dicha automatización.

Se justificó con exactitud la viabilidad del proyecto propuesto a la compañía, de manera que al realizar el balance costo-beneficio se evidencia la puesta en marcha de la automatización propuesta sin temor a incurrir en inversiones innecesarias o irrecuperables. 
Se realizó un estudio minucioso del área a automatizar de manera que se propuso de forma específica el equipamiento requerido y así evitar que sobre o falte instrumentación, en cuanto a cantidad y capacidad, de acuerdo a la automatización deseada para esta área de la compañía.

Se establece la propuesta de automatizar el área final de embalaje, selección, inventariado y paletizado del producto terminado en la empresa, de forma que se pueda llevar al mínimo la intervención humana en dicha etapa y evitar los esfuerzos anti-ergonómicos del personal involucrado.

Se propuso específicamente la implementación de sensores, actuadores, software e instrumentación requerida, para lograr la separación, inventariado y distribución de los siete diferentes productos que la empresa produce, según sus códigos de barras; y así lograr que los hombres no hagan el trabajo que fácilmente pueden hacer las máquinas.

\section{Desarrollo}

\section{Estado actual}

El proceso que se propuso automatizar, o mejor dicho convertirlo en un proceso semi-automático, en Kimberly Clark, planta de San Antonio, Belén, se encuentra en la bodega de producto terminado, área de entarimado. Esta bodega es la parte final del proceso productivo, acá es el punto donde convergen todas las diferentes líneas productivas de la planta, dicha bodega es un lugar pequeño el cual tiene capacidad para almacenar la producción de un día y medio, desde acá el producto es enviado al Centro de Distribución con el que cuenta la empresa en Desamparados, San José o los diferentes centros de distribución en Centro América y República Dominicana.

En la bodega de producto terminado se cuenta con un área de entarimado, zona donde se acomoda el producto terminado en la correspondiente tarima antes de pasar a ser almacenadas, esta zona labora 6 días a la semana 24 horas, contando con tres cuadrillas de personal que trabajan en turnos de 8 horas; dichas cuadrillas están formadas por 4 entarimadores y 1 supervisor. A la zona de entarimado llega la producción de las 7 líneas de producción, cada producto es tomado indistintamente por un entarimador y llevado a la tarima correspondiente, este proceso se hace completamente manual, cada entarimador toma el producto y lo coloca en alguna de las 7 tarimas que se encuentra a lo largo de la zona, esto hace que cada uno de ellos en su turno de 8 horas camine alrededor de 2 kilómetros, tomando en cuenta que la producción es de aproximadamente 5000 bultos por turno, y cada uno de ellos con un peso aproximado de 8 kilos, lo hace un trabajo muy desgastante. Adicionalmente, en esta parte del proceso el supervisor del área tiene la responsabilidad de elaborar una boleta con la cantidad de producto que lleva cada tarima, 
registrarlo y hacer cortes cada 30 minutos, los cuales son anotados en una base de datos de Excel para que pueda ser consultada por las líneas y conozca el saldo de su producción. La figura 1 muestra el estado actual del área en mención.

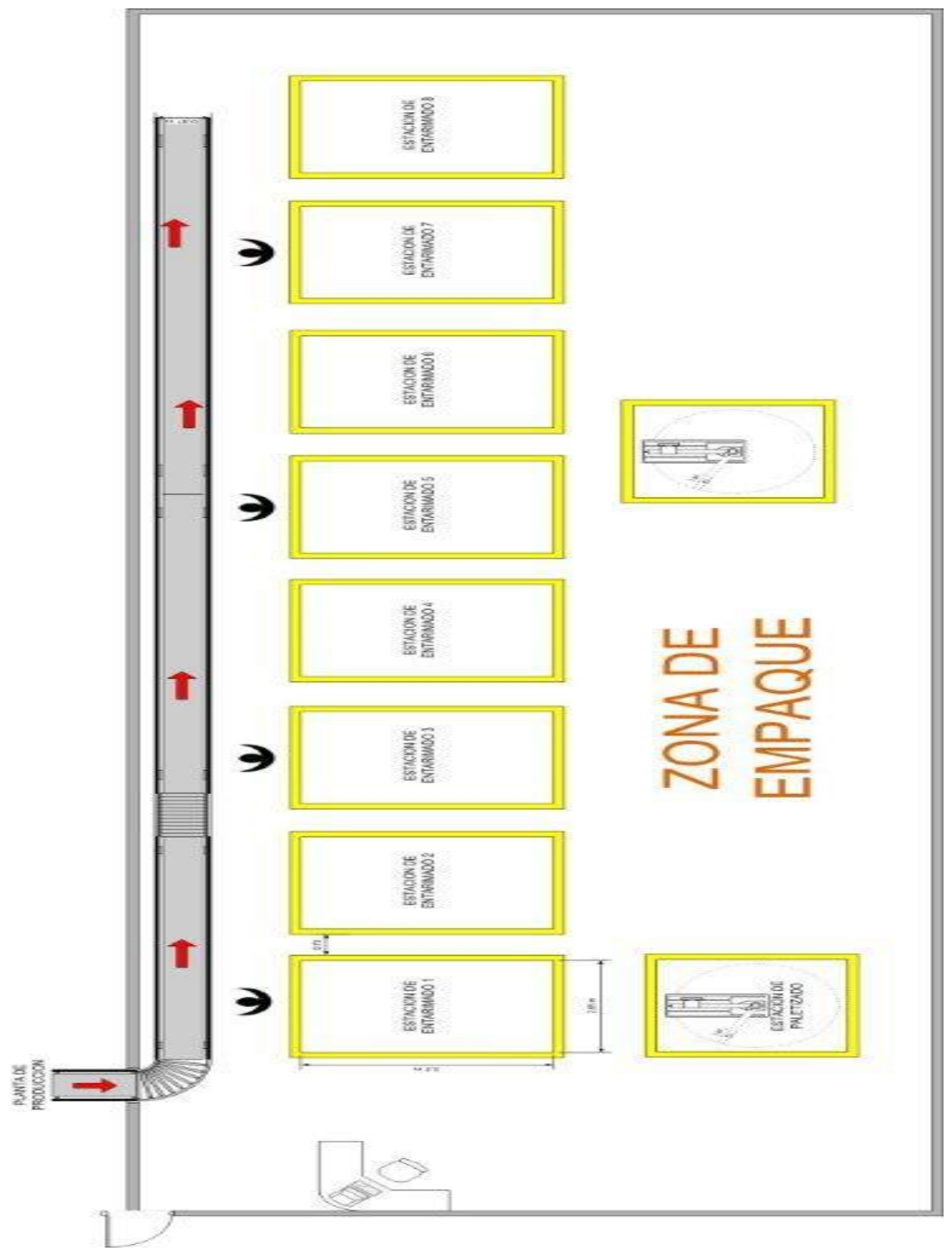

FIGURA 1. Diagrama estado actual área a automatizar 
El trabajo diario involucra mucho la operación manual y de una forma inapropiada para el trabajador por los movimientos tan mecánicos y rutinarios. El área cuenta con dos conveyors de rodillos ( 1 a la entrada a la bodega y otro después de la primera banda transportadora), los cuales tienen la función de transportar y disminuir la velocidad con la que ingresa el producto. También cuenta con 3 bandas transportadoras, la primera se encuentra entre los dos conveyors de rodillo. Adicionalmente hay 2 máquinas paletizadoras que tienen la función de colocar el plástico alrededor de la tarima y el producto terminado para su protección. Estas paletizadoras no tienen costo para la empresa ya que son propiedad del proveedor del plástico el cual las da como parte del servicio por la compra del material.

\section{Propuesta y estado final}

Basado en el análisis de la situación imperante en el área estudiada, se propuso adquirir e instalar una serie de accesorios para automatizar el área y así hacer más eficiente y más amigable con el operador, dicha área.

Cada etapa de la propuesta fue seguida por asesoría de expertos de la propia empresa, de manera que se seleccionaron los sensores, actuadores y dispositivos en general, que más se ajustaran a las necesidades de la empresa en el área a automatizar.

Una vez que se instale la automatización propuesta a la empresa en su área de bodega y entarimado, se contará con los siguientes dispositivos adicionales a lo que hay actualmente en funcionamiento, los cuales se detallan junto con sus funciones a continuación:

- Un lector de código de barras para monitoreo de los siete diferentes productos que la empresa produce

- Dos pistones en diferentes tramos de la banda para que coloquen el producto en la respectiva banda de descarga

- Una compuerta donde va cada pistón (dos) que impidan o permitan el paso del producto según corresponda

- Dos sensores de presencia que indiquen cuando accionar el pistón y la compuerta respectiva.

- Tres bandas de descarga por donde saldrán los siete productos a ser entarimados según su código de barras. Para la última banda no se necesitará pistón ni compuerta porque los productos que no accionan ninguno de los pistones previos, automáticamente caerán en esta banda. Dos bandas descargaran dos productos y una banda tres productos. 
- Una paletizadora más, para que queden tres en total; una al final de cada banda de descarga. Esta es donada por los proveedores del material de empaque (plástico), por lo tanto no se incurrirá en gastos por la adquisición de la misma.

- El software para la programación del hardware automáticamente y el manejo automático del inventario de producto terminado.

\section{Descripción del funcionamiento con la automatización propuesta}

El producto terminado sale en orden aleatorio por la banda del túnel de arribo, proveniente del área de producción, luego pasa por los rodillos en la curva que ubica el producto en la banda final, antes de la cual se colocará el lector de código de barras que enviará la señal para indicar por cuál banda de descarga deberá salir dicho producto, así que, cerrará la compuerta y accionará el pistón correspondiente en el momento en que el sensor detecte la presencia de la caja o bulto de producto, o si es el caso que deje pasar el producto hasta la última banda de descarga según corresponda. Una vez colocado el producto en la respectiva banda de descarga este es transportado por un trayecto aproximado de dos metros donde es tomado manualmente por el operador y colocado en las tarimas correspondientes y posteriormente paletizado y colocado con el montacargas en la bodega. El lector de código de barras permitirá llevar el inventario de producto terminado totalmente ágil, automático y accesible en tiempo real, lo cual evita el error humano en el conteo y permite la eliminación o reubicación de dos de las plazas, por turno (tres turnos) de operación de la planta (el supervisor y 1 entarimador).

El proceso será controlado por medio de un sistema DCS proveído por la compañía FoxBoro, en este caso la empresa no tiene que realizar inversión en el mismo, ya que cuenta con él y tiene el personal especializado en la programación del mismo.

Con todo esto, se logrará tener una distribución más ágil y eficaz del producto terminado en el área de bodega y entarimado, logrando alcanzar que los empleados de dicha área tengan una adecuada ergonomía en el desempeño de sus trabajos y no tengan que estar haciendo movimientos rutinarios, cansados por desplazamientos inadecuados y desgastantes por pesos excesivos en el acumulado al final de su jornada.

Se logrará reducir el número de plazas que operan dicha área, pasando de seis personas por turno en la actualidad a cuatro personas por turno cuando se implemente la propuesta si se hace efectiva. Lo que ahorraría el equivalente a los salarios de seis operadores y permitiría un retorno de inversión acelerado, o en su defecto abre la posibilidad de reubicar seis operarios a otras áreas de la empresa, donde requieran más mano de obra. 
Una de las grandes ventajas de la compañía es que cuenta con personal altamente calificado, con vasta experiencia en temas de automatización, a tal punto que ellos mismos se dan la asistencia y mantenimiento en las otras áreas de la planta que ya cuentan con procesos con mayor automatización. Además no requieren frenar ningún proceso para implementar dicha propuesta ya que los accesorios se irán implementando sin alterar el funcionamiento diario del área y de ser necesario un día sin producción, se realizaría un día no hábil para no frenar ningún proceso.

A continuación presentamos en la figura 2, el diagrama de la situación final esperada, luego de la automatización propuesta, si ésta es implementada por la empresa Kimberly Clark en San Antonio de Belén, en el área de entarimado y bodega. 


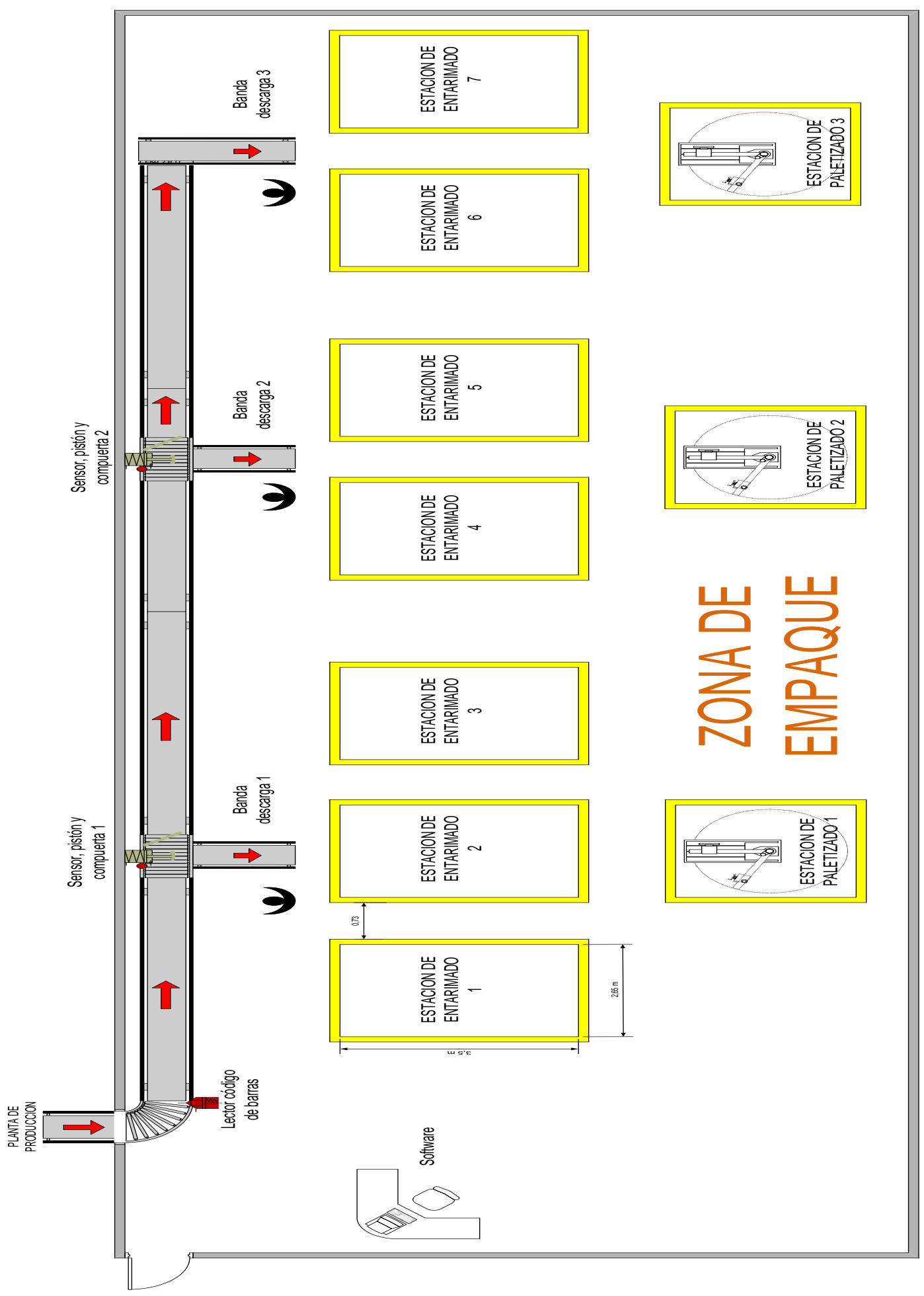

FIGURA 2. Diagrama estado final de la automatización 


\section{Análisis de costos del proyecto}

Según se muestra en el cuadro 1, los costos de implementación de la automatización propuesta a Kimberly Clark son bastantes razonables, ya que no superan los $\$ 15000$ de inversión.

CUADRO 1. Costos de accesorios para automatización

\begin{tabular}{|c|c|c|c|}
\hline Descripción & Cantidad & $\begin{array}{l}\text { Costo } \\
\text { (\$)/unidad }\end{array}$ & Total \\
\hline Sensor & 2 & $\$ 130$ & $\$ 260$ \\
\hline Lector de Código de barras & 1 & $\$ 500$ & $\$ 500$ \\
\hline Conveyors y pistón & 3 & $\$ 1500$ & $\$ 4500$ \\
\hline Fibra Óptica & 50 & $\$ 85$ & $\$ 4250$ \\
\hline Software Lector de código & 1 & $\$ 5000$ & $\$ 5000$ \\
\hline & & TOTAL & $\$ 14510$ \\
\hline
\end{tabular}

El cuadro 1, presenta la descripción y cantidad de los insumos necesarios para la ejecución de la mejora proyectada, con sus respectivos costos. Una vez analizado el costo se debe considerar el ahorro que generará el proyecto, como se ha mencionado este ahorro es básicamente el salario de los dos operarios menos, que no se necesitarían más en el área de empaque.

La figura 3, muestra la estimación del ahorro por concepto de mano de obra el cual ronda los $\$ 4$ 600 por mes, además de las cargas sociales por el mismo concepto, en caso de prescindir de los servicios de los funcionarios. 


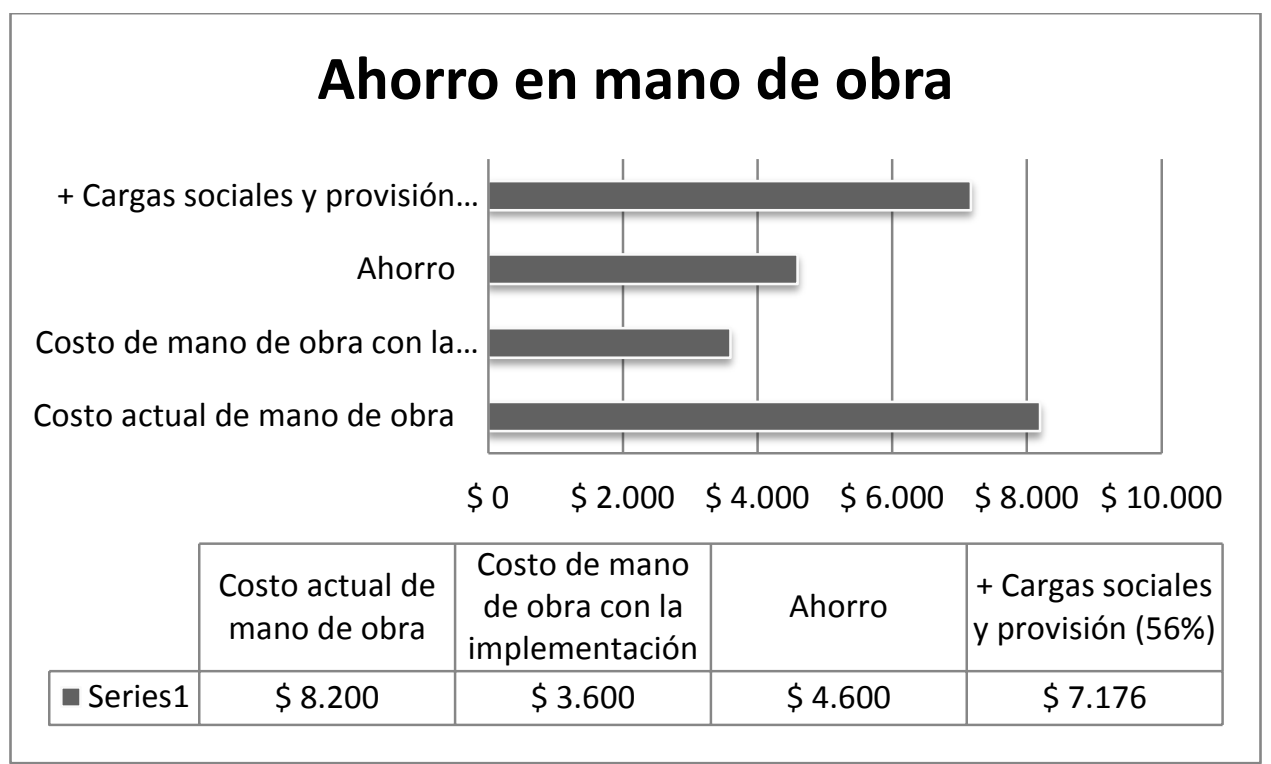

FIGURA 3. Ahorro por concepto de mano de obra en $\$ /$ mes

Considerando el ahorro de mano de obra como el principal beneficio económico del proyecto y además tomando en cuenta que no existen costos adicionales de mantenimiento del equipo, pues ese mantenimiento es realizado por personal de planta de la empresa, a continuación la figura 4, muestra el balance costo-beneficio proyectado a cinco meses desde la implementación de lo propuesto.

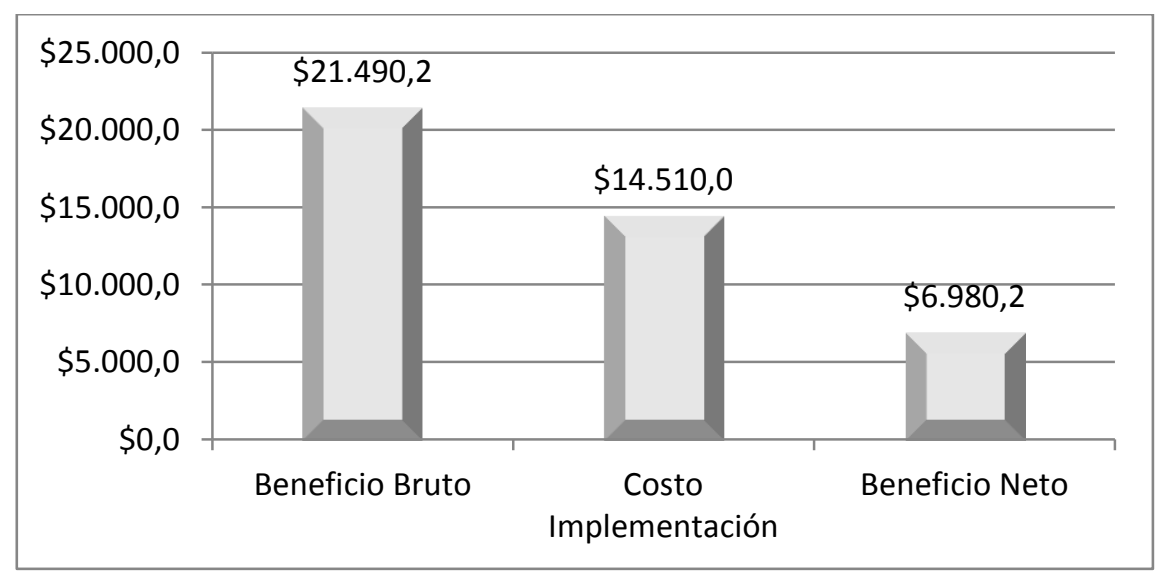

FIGURA 4. Balance costo/beneficio proyectado a cinco meses

La figura anterior evidencia como en 5 meses se obtendría un beneficio neto de casi $\$ 7$ mil lo cual permite recuperar la inversión inicial en un lapso muy corto de tiempo, tal y como se indica en el cuadro 2 , donde se muestra el cálculo del retorno sobre la inversión, el valor actual neto de la 
inversión y el tiempo de recuperación de la misma, junto con el desglose por mes de lo presentado en la figura 4.

El cálculo se realiza utilizando una tasa de descuento del $10 \%$ que es el estándar utilizado por la compañía para este tipo de proyectos.

CUADRO 2. Beneficios económicos de la inversión

\begin{tabular}{|l|c|c|c|c|c|c|c|}
\hline Descripción & Mes 0 & Mes 1 & Mes 2 & Mes 3 & Mes 4 & Mes 5 & TOTAL \\
\hline Beneficio Bruto & 0 & $\$ 5,023$ & $\$ 5,249$ & $\$ 5,485$ & $\$ 5,732$ & $\$ 5,990$ & $\$ 21,490$ \\
\hline $\begin{array}{l}\text { Costo } \\
\text { Implementación }\end{array}$ & $\$ 14,510$ & $\$ 0$ & $\$ 0$ & $\$ 0$ & $\$ 0$ & $\$ 0$ & $\$ 14,510$ \\
\hline Beneficio Neto & $-\$ 14,510$ & $\$ 5,023$ & $\$ 5,249$ & $\$ 5,485$ & $\$ 5,732$ & $\$ 5,990$ & $\$ 6,980$ \\
\hline TIR & \multicolumn{7}{|c|}{$\mathbf{2 5 \%}$} \\
\hline VAN & $\$ 3,884.13$ \\
\hline $\begin{array}{r}\text { Tiempo de } \\
\text { Recuperación }\end{array}$ & \multicolumn{7}{|c|}{$\mathbf{2 . 8 9}$ meses } \\
\hline ROI & \multicolumn{7}{|c|}{0.48} \\
\hline
\end{tabular}

Como se observa en el cuadro 2, el proyecto tienen una tasa interna de retorno (TIR) del $25 \%$ apenas a los 5 meses lo cual es un excelente rendimiento para un proyecto de automatización, además el valor actual neto (VAN) a 5 meses después de la implementación sería de $\$ 3884$, lo cual es una inversión bastante baja para el beneficio que traería el proyecto como tal.

Por otro lado el tiempo de recuperación de la inversión es de apenas 2,89 meses, un tiempo sumamente corto para recuperar una inversión de $\$ 15000$, incluso el rendimiento sobre la inversión (ROI) de 0,48 a 5 meses es muchísimo más alto que la tasa básica pasiva que pagan los bancos en Costa Rica por las inversiones que es de apenas un 0.08, lo cual es otro indicador de que la inversión es más que rentable.

De esta forma se evidencia lo apropiado de un proyecto de automatización de esta índole por todo lo que llegaría a significar para la empresa involucrada.

\section{Consideraciones finales}

- El alcance de la automatización va más allá de una simple mecanización de los procesos ya que ésta provee a operadores humanos mecanismos para asistirlos en los esfuerzos 
físicos del trabajo, la automatización reduce ampliamente la necesidad sensorial y mental del humano. De manera que con la automatización se busca que las máquinas hagan trabajo de máquinas, pero que los hombres no tengan que hacer lo que las máquinas pueden hacer.

- Con la automatización propuesta a la empresa, se logrará tener una distribución más ágil y eficaz del producto terminado en el área de bodega y entarimado, logrando alcanzar que los empleados de dicha área tengan una adecuada ergonomía en el desempeño de sus trabajos y no tengan que estar haciendo movimientos rutinarios, cansados por desplazamientos inadecuados y desgastantes por pesos excesivos en el acumulado al final de su jornada.

- Otro punto alto de la automatización propuesta, es que se lograría reducir el número de plazas que operan dicha área, pasando de seis personas por turno en la actualidad a cuatro personas por turno cuando se implemente la propuesta si se hace efectiva. Lo que ahorraría el equivalente a los salarios de seis operadores y permitiría un retorno de inversión acelerado (en tan solo tres meses), o en su defecto abre la posibilidad de reubicar seis operarios a otras áreas de la empresa, donde requieran más mano de obra.

- Desde el punto de vista financiero, que es uno de los rubros que más cuidan las empresas; de acuerdo con los análisis realizados, evidencian una inversión muy baja para la cantidad de beneficios que traería a la empresa esta propuesta.

- Se demostró como la inversión será recuperada en un tiempo mínimo y el ambiente laboral será mucho más amigable para los empleados del área, lo que definitivamente permite ver que es un proyecto bien pensado, que se propuso pensando en mejorar las condiciones para todas las partes, de manera que la gran beneficiada sea toda la compañía.

- Es claro que los procesos de automatización no representan un gasto para las empresas como en algunos casos se piensa, si no, que las mejoras en eficiencia, tiempos de respuesta y calidad, convierten esos gastos en inversiones altamente productivas y rentables para la compañía.

\section{REFERENCIAS}

Anónimo. (s.f.). Recuperado de

http://www.ing.uc.edu.ve/ emescobar/automat I/contenido menu/Unidad I/Contenido/pagina3 /pagina3.htm

Arbones, E. (2009). Técnicas Gráficas en Prodúctica. España: Marcombo. Recuperado de http://site.ebrary.com/lib/sibdilibrosp/reader.action?doclD=10337617\&ppg=4 
Craig, J. (2006). Robótica. Pearson Educación.

Daneri, P. (2008). PLC: Automatización y Control Industrial. Editorial Hispano Americana HASA. Recuperado de

http://site.ebrary.com/lib/sibdilibrosp/reader.action?doclD=10336954

Domingo, J. (2003). Introducción a los Autómatas Programables. Editorial UOC.

Gallardo, S. (2013). Técnicas y Procesos en Instalaciones Domóticas y Automáticas. Ediciones Paraninfo, S.A.

García, A. (2005). El Control Automático en la Industria. Universidad de Castilla La Mancha.

Hernández, P. (2012). Guía Práctica de Rapid Manufacturing. España: Universidad de Las Palmas de Gran Canaria. Servicio de Publicaciones y Difusión Científica. Recuperado de http://site.ebrary.com/lib/sibdilibrosp/reader.action?doclD=10732596\&ppg=24

Pardo, J. (2012). Montaje y Puesta en Marcha de Sistemas Robóticos y Sistemas de Visión en Bienes de Equipo y Maquinaria Industrial (UF0461). España: IC Editorial. Recuperado de http://site.ebrary.com/lib/sibdilibrosp/reader.action?doclD=10721454

Rodríguez, A. (2008). Comunicaciones Industriales. España: Marcombo. Recuperado de http://site.ebrary.com/lib/sibdilibrosp/reader.action?doc/D=10232353\&ppg=10

Rodríguez, J. (2014). Automatismos industriales. Ediciones Paraninfo, S.A. 